\title{
La sensibilité vésicale et son évaluation
}

\section{Bladder sensitivity and its evaluation}

\author{
G. Amarenco - M. Jousse \\ (C) Springer-Verlag 2009
}

Parmi les symptômes urinaires habituellement rencontrés en pratique, les signes « irritatifs » sont d'une grande fréquence. Urgences mictionnelles, pollakiuries diurnes et/ou nocturnes, fuites sur impériosité, nocturie, sont des motifs de consultation très habituels tant chez l'homme que chez la femme. L'attitude habituelle après avoir éliminé une étiologie infectieuse (ECBU) et urologique (cystoscopie) est d'essayer un traitement d'épreuve par anticholinergiques et, en cas d'échec, de pratiquer un bilan urodynamique pour mieux en préciser les mécanismes physiopathologiques et étiopathogéniques. Ces explorations retrouvent parfois une anomalie motrice du détrusor lors de la phase de remplissage sous la forme de contractions non inhibées entrant dans le cadre des vessies hyperactives. Ailleurs, le bilan, même en conditions «sensibilisatrices » (position debout, remplissage rapide, test à l'eau glacé, holter vésical), s'avère négatif, et il est alors parfois commode d'évoquer non plus un trouble moteur mais une altération sensitive vésicale ou urétrovésicale. L'existence d'un besoin trop «précoce », une capacité de remplissage limitée par une franche hyperesthésie sont des éléments qui peuvent entrer dans ce syndrome.

Il est pourtant toujours bien difficile de mesurer objectivement une sensation purement subjective (le «besoin d'uriner »), parfois même de la faire verbaliser correctement au patient [1]. Quelles circonvolutions sémantiques ne doit-on pas souvent prendre pour expliciter la différence entre un besoin « habituel », « pressant », « urgent », « impérieux »! Parfois même le subtil distinguo entre «sensation de remplissage conscient », de « pesanteur » et «d'envie » est bien difficile... Tout cela rend bien problématique la quantification et bien aléatoire la reproductibilité de l'analyse des sensations à point de départ vésical.

Plusieurs approches sont ainsi possibles dans l'évaluation de la sensibilité vésicale : une approche purement clinique

G. Amarenco $(\bowtie) \cdot$ M. Jousse

Service de rééducation neurologique et d'explorations périnéales, hôpital Rothschild, 33, boulevard de Picpus,

F-75571 Paris cedex 12, France

e-mail : gerard.amarenco@rth.aphp.fr et une approche instrumentale, qu'elle soit manométrique, psychophysiologique ou électrophysiologique.

\section{Approche clinique}

Elle est fournie par les données du catalogue mictionnel.

De Wachter et Wyndaele ont montré, en 2003, que les catalogues mictionnels avec recueil des sensations vésicales permettaient de recueillir les mêmes sensations de besoins pour les mêmes volumes que pendant le remplissage cystomanométrique chez des sujets volontaires sains [2]. Naemova et al. montrèrent en 2008 que les volumes vésicaux pendant la cystomanométrie étaient supérieurs à ceux du catalogue mictionnel pour des sensations vésicales correspondantes chez des patientes incontinentes [3]. Mais ces auteurs ont utilisé, pour quantifier l'intensité des sensations vésicales, l'échelle suivante :

- absence de désir d'uriner ;

- première sensation de besoin, mais la miction peut être différée de 30 minutes (min) ;

- fort besoin d'uriner, et la miction ne peut être différée de plus de 15 minutes;

- besoin urgent d'uriner.

Cette échelle qualitative décrit un besoin non pas progressif et linéaire mais par paliers, ne semblant pas correspondre aux sensations vésicales physiologiques. En effet, selon Dompeyre et al., les sensations vésicales pendant la cystomanométrie seraient une même sensation qui augmenterait pendant le remplissage continuellement et de façon linéaire après une phase de latence, mesurable sur une échelle visuelle analogique [4].

\section{Approche « urodynamique »}

Elle est très classique et fort ancienne : c'est le volume de remplissage noté lors des sensations de remplissage et de besoin signalées par le patient au cours de la cystomanométrie à différentes vitesses de remplissage et à différentes 
températures. La version française de la validation de la terminologie effectuée par l'International Continence Society (ICS) décrit ainsi les sensations vésicales normales survenant au cours d'une cystomanométrie [1] : première sensation de remplissage, premier besoin d'uriner et fort besoin d'uriner. Mais il ne s'agit pas de conditions physiologiques. En effet, l'examen se déroule en position semi-assise, alors que l'homme est un être vertical, avec des sondes modifiant les récepteurs sensitifs et les voies afférentes, avec un stimulus non physiologique, avec de l'eau de compositions chimique et thermique différentes de l'urine, un remplissage vésical rétrograde et un débit de remplissage souvent supérieur au débit physiologique estimé à $1 \mathrm{ml} / \mathrm{kg}$ par heure. La reproductibilité immédiate et à court terme n'est pas bonne ; par contre, elle s'améliore sur le long terme. Il semble que la première cystomanométrie influence les sensations lors d'une seconde cystomanométrie. D'autres auteurs pensent que ces sensations au cours de la cystomanométrie ne correspondent pas à la sensation de remplissage et de besoin, mais pourraient être dépendantes de la sensation de cathéter intra-urétral ou d'un autre facteur.

\section{Analyse psychophysiologique}

Au cours d'une cystomanométrie, elle a été évaluée au moyen d'un microclavier électronique gradant en cinq points la sensation de besoin, et ce, d'une manière continue par le patient préalablement informé, sans aucune intervention du médecin en termes de questionnement sur le besoin. Cette technique s'est avérée parfaitement reproductible, avec de plus une parfaite corrélation entre le niveau de sensibilité et le degré de remplissage vésical.

Ces études de la perception du, ou plutôt des besoin(s), au cours de la cystomanométrie, sont certes le moyen d'évaluation le plus classique, mais elles requièrent toutefois une totale participation du patient, avec un dialogue permanent entre patient et technicien, nécessitant ainsi une compréhension parfaite des événements prédits (première sensation de besoin d'uriner, sensation de besoin habituel, sensation de besoin urgent [classification ICS], ne devant pas être involontairement ou... volontairement confondus avec la perception consciente du remplissage ou avec l'existence d'un inconfort urétrovésical généré par la présence de la sonde). La reproductibilité est donc très imparfaite, et la fiabilité de la méthode peu encourageante dans le cadre d'une évaluation protocolée. L'utilisation de dispositif électronique reste toujours d'interprétation très délicate, en raison de plusieurs facteurs : caractère non physiologique du remplissage (tant en termes de vitesse de remplissage que de voie..., d'administration par cathéter transuréthral pouvant singulièrement modifier la perception sensitive habituelle); anxiété et/ou inhibition du patient pendant l'examen; parfois température non physiologique du liquide perfusé. En revanche, l'utilisation du dispositif électronique permet de s'affranchir de la présence et du questionnement constant (possiblement inducteur de réponses inappropriées) du médecin.

L'analyse au cours d'une cystométrie, d'une part, des réponses cutanées sympathiques sur la paume et la plante et, d'autre part, de l'activité électromyographique des muscles périnéaux à l'aide d'une électrode de surface peut être une alternative. Au moment du premier besoin, apparaissent de manière concomitante des décharges de potentiels cutanés sympathiques et une majoration de l'activité électromyographique périnéale.

Cependant, l'apparition d'une réponse cutanée sympathique (réponse électrodermique, potentiel cutané sympathique, réflexe psychogalvannique...) est totalement aspécifique. Les potentiels évoqués cutanés sympathiques sont en effet le fait d'une variation de résistance des tissus cutanés induite par la stimulation des glandes sudoripares, secondaire à l'activation des fibres non myélinisées de type $\mathrm{C}$ des nerfs sympathiques efférents qui innervent ces glandes. Cette activation des voies végétatives sympathiques peut être induite par n'importe quels stimuli «éveillant» qu'il s'agisse de stimulations sonores, électriques (ce qui permet de mesurer une latence), cognitive (principe du détecteur de mensonges)... et une puissante sensation physiologique comme la peur, la surprise, le froid... et le besoin d'uriner !

L'utilisation pratique semble assez limitée aux «simulateurs » (dans un sens ou dans l'autre) qui décrètent ne pas sentir le besoin (qui serait alors détecté par l'apparition de bouffées de potentiels sympathiques) ou qui assurent le sentir d'emblée...

Un autre moyen instrumental est l'évaluation de la sensibilité vésicale, l'étude des potentiels évoqués par remplissages vésicaux répétés, mais quand on connaît le nombre de pathologies dont le substratum physiopathologique est un trouble de la sensibilité, cette technique reste d'être mise au point et validée... chez l'homme.

L'étude des seuils de perception d'une stimulation électrique peut être utilisée, mais il semble que ces seuils dépendent de la forme du courant appliqué (onde sinusoïde ou carrée), de l'état de remplissage vésical, de la position exacte des électrodes au contact de la paroi vésicale, ce qui rend cette technique difficilement reproductible et comparable selon les études.

Récemment, l'imagerie fonctionnelle a permis d'établir plusieurs régions impliquées dans la sensation de remplissage vésical (la substance grise périaqueducale, le pont, le cortex cingulaire, le gyrus frontal moyen droit et inférieur droit cortex pariétal droit et gauche, les lobes cérébelleux latéraux droit et gauche) et le besoin d'uriner (les aires prémotrices droites et gauches ainsi que l'hypothalamus). 
Ainsi, à ce jour, la nécessaire évaluation de la sensibilité vésicale reste peu objective. Les travaux futurs devraient pouvoir se focaliser sur ce type d'évaluation, en raison de la fréquence des pathologies impliquées et de leurs conséquences thérapeutiques.

\section{Références}

1. Haab F, Amarenco G, Coloby P, et al (2004) Terminology of lower urinary tract dysfunction: French adaptation of the terminology of the International Continence Society. Prog Urol 14(6):1103-11

2. De Wachter S, Wyndaele JJ (2003) Frequency-volume charts: a tool to evaluate bladder sensation. Neurourol Urodynam 22: 638-42

3. Naoemova I, Van Meel T, De Wachter S, Wyndaele JJ (2008) Does sensory bladder function during cystometry differ from that in daily life? A study in incontinent women. Neurourol Urodynam 28(4): 309-12

4. Dompeyre P, Fritel X, Bader G, et al (2007) Bladder sensitivity testing using a visual analogue scale: comparative cystometric study on women. Neurourol Urodynam 26:350-5

\section{基 Springer}

the language of science

\section{springer.com}

\section{Sign up for SpringerAlerts}

The best way to keep you up-to-date with new developments in your field!

You can customize your SpringerAlerts to deliver exactly the information you need!

We offer

- Table of Contents Alerts for Journals

- Table of Contents Alerts for Book Series

$\rightarrow$ New Book Alert

As an alerts subscriber, you will receive

- Reliable news about journals and upcoming books

- Special offers - be the first to know about free online access

to journals and discounts on books

springer.com/alerts - fast, free and flexible 